\title{
Large volume cryoprotectant-free vitrification: an alternative to conventional cryopreservation for human spermatozoa
}

\author{
M. Slabbert ${ }^{1}$, S.S. du Plessis ${ }^{2}$ \& C. Huyser ${ }^{1}$ \\ 1 Department of Obstetrics and Gynaecology, University of Pretoria, Steve Biko Academic Hospital, Pretoria, South Africa; \\ 2 Division of Medical Physiology, Department of Biomedical Sciences, Stellenbosch University, Cape Town, South Africa \\ Correspondence \\ Marisa Slabbert, Reproductive Biology Laboratory, Department of Obstetrics and Gynaecology, University of Pretoria, Steve Biko Academic \\ Hospital, Pretoria, \\ South Africa. \\ Tel.: +27 12354 2062/2067; \\ Fax: +27 123296258 ; \\ E-mail: marisa.slabbert@gmail.com
}

\section{Summary}

Vitrification is a simple and cost-effective method for the storage of human spermatozoa without the use of conventional cryoprotectants, by plunging the sperm suspension directly into liquid nitrogen. As a result, solidification of living cells without the formation of ice crystals is achieved during cooling. This study aimed to compare cryoprotectant-free vitrification to conventional cryopreservation protocols. Semen samples $(n=35)$ were collected from patients seeking diagnostic assistance at the Reproductive and Endocrine Unit at Steve Biko Academic Hospital. Samples were processed using a discontinu-ous density-gradient centrifugation method. Washed samples were split into two aliquots and cryopreserved either by means of cryoprotectant-free vitrifica-tion (sucrose $+1 \%$ albumin) or conventional slow freezing (TEST-yolk buf-fer). Post-thawing, the sperm motion parameters, mitochondrial membrane potential $(\Delta \psi \mathrm{m})$ and DNA fragmentation were compared between the two groups. No significant differences were observed in the sperm motility parame-ters $(P>0.05)$. Significantly higher percentages of $\Delta \psi \mathrm{m}(11.99 \% \pm 4.326 \%$ versus $6.58 \% \pm 1.026 \% ; P<0.001)$ and lower percentages of DNA fragmenta-tion $(2.79 \% \pm 1.017 \%$ versus $3.86 \% \pm 1.38 \% ; P<0.01)$ were observed when comparing cryoprotectant-free vitrification to conventional cryopreservation. Cryoprotectant-free vitrification is a rapid and promising alternative to conventional methods resulting in good-quality spermatozoa post-thaw.

\section{Keywords}

Cryopreservation—DNA integrity—human spermatozoa—mitochondrial membrane potential—vitrification

\section{Introduction}

Cryobiology is a fast-evolving field, with promising applications in reproductive biology (Isachenko et al., 2012a). Temperatures below subzero together with appropriate cryoprotective agents (CPA) preserve the physiological and reproductive functions of cells, making long-term storage without the associated loss of viability possible. An alternative preservation method as opposed to conventional cryopreservation protocols is vitrification. This method preserves the cells by plunging the suspension directly into liquid nitrogen. As a result, solidification of living cells without the formation of ice crystals is achieved during cooling (Isachenko et al., 2004a; Vutyavanich et al., 2010). The described method for vitrification of oocytes, embryos and other tissues requires rapid cooling rates and high CPA concentrations (Isachenko et al., 2005). Spermatozoa have low tolerance levels for high concentrations of cryoprotectants with these agents causing possible lethal osmotic effects and chemical alterations in spermatozoa (Isachenko et al., 2003). Vitrification without the use of conventional CPA has therefore been described for human spermatozoa (Isachenko et al., 2003, 2004a,b). This method requires replacing the CPA with a protein and carbohydrate solution (1\% human serum albumin [HSA] and $0.25 \mathrm{M}$ sucrose). A major disadvantage of cryoprotectant-free vitrification was that only small volumes of spermatozoa $(\leq 20 \mu \mathrm{l})$ could be vitrified. In addition, most of the proposed methods were described as open systems and did not prevent direct contact with liquid nitrogen (Isachenko et al., 2003, 2004a, 2005). Different aseptic vitrification techniques were investigated by Isachenko et al. (2005); however, only small volumes, ranging between 1 and $40 \mu \mathrm{l}$, of sperm suspension could be vitrified in these systems. In 2011, the Isachenko group reported a novel 
aseptic cryoprotectant-free vitrification method allowing for the vitrification of larger volumes (up to $500 \mu \mathrm{l}$ ) of spermatozoa (Isachenko et al., 2011, 2012a).

This study aimed to compare the effects of conventional slow freezing versus cryoprotectant-free vitrification, described by Isachenko et al. (2012a) with regard to motility parameters, mitochondrial membrane potential $(\Delta \psi \mathrm{m})$ and DNA integrity of washed human spermatozoa.

\section{Materials and methods}

Institutional approval for this study was received from Steve Biko Academic Hospital (SBAH) and the Research Ethics Committee of the University of Pretoria (protocol number S48/2012). Informed consent was received from all participants.

\section{Semen preparation}

Semen samples $(n=35)$ from healthy, HIV-negative patients (18-35 years), without any pathologies, seeking diagnostic assistance from the Reproductive Biology and Endocrine Unit, SBAH, were included in the study. The inclusion criteria were a minimum concentration of $15 \times 10^{6}$ sperm ml ${ }^{-1}$, total sperm motility of at least $40 \%$ and a minimum semen volume of $1.5 \mathrm{ml}$. All semen samples complied with the minimum criteria of the World Health Organization (WHO) (2010). The average preprocessed values of the sample population included in the study were as follows: semen volume $2.98 \mathrm{ml}( \pm 1.071)$, concentration $41.87 \times 10^{6}$ sperm ml ${ }^{-1}( \pm 17.81)$, total motility $74.47 \%( \pm 9.02)$ and sperm morphology $9.77 \%$ $( \pm 2.46)$. Semen analyses were performed according to the standard operating procedures of the Unit based on the WHO (2010) guidelines for the examination and processing of human semen. Each ejaculate was processed by means of a discontinuous gradient centrifugation method, according to the manufacturer's guidelines (Nidacon $^{\text {TM }}$ International, Mölndal, Sweden, PureSperm 40/80. Available from http://nidacon.com/products/puresperm-4080/). After the final wash step, the supernatant was discarded; the pellet was resuspended and split into two aliquots for (i) conventional slow freezing and (ii) cryoprotectant-free vitrification.

\section{Conventional slow freezing}

Freezing medium-TYB (Test yolk buffer; Irvine Scientific ${ }^{\circledR}$, Santa Ana, CA, USA) was added to the washed spermatozoa in a $1: 1$ ratio. The sperm suspension was aspirated into $0.5 \mathrm{ml} \mathrm{CBS}^{\mathrm{TM}}$ straws (Cryo Bio System, Paris, France), and both ends were sealed twice hermetically using an alcohol flame burner and forceps. The loaded straws were then kept at room temperature for $10 \mathrm{~min}$. The manufacturer's protocol was adapted by excluding the refrigeration period. Subsequent to the room temperature incubation, straws were placed horizontally in the vapour phase for $15 \mathrm{~min}$ and then submerged into liquid nitrogen. Straws were stored in a liquid nitrogen tank (Thermo Fisher Scientific, Johannesburg, South Africa) for at least $24 \mathrm{~h}$.

For thawing, cryopreserved straws were immersed in water $\left(23^{\circ} \mathrm{C}\right.$ ) for $5 \mathrm{~min}$ (according to the manufacturer's instruction). Hereafter, the straws were sterilised using $70 \%$ alcohol and dried, and the ends were cut open. The contents were expelled into a centrifuge tube (BD Pharmingen $^{\mathrm{TM}}$, Franklin Lakes, NJ, USA) containing $2 \mathrm{ml}$ PureSperm ${ }^{\circledR}$ Wash (Nidacon ${ }^{\mathrm{TM}}$ International) and centrifuged for $5 \mathrm{~min}$ at $300 \mathrm{~g}$. The supernatant was removed; the pellet was resuspended and post-sperm counts were performed.

\section{Cryoprotectant-free vitrification for larger volumes}

Results from a pilot study found no significant differences in the total motility $[\mathrm{a}+\mathrm{b}: \quad 29.4 \% \pm 9.6$ versus $31.8 \% \pm 10.3, P=0.546$ ], rapid progressive motility [a: $20.6 \% \pm 10.07$ versus $22.1 \% \pm 10.58, P=0.546]$ or viability (Annexin V/PI apoptosis assay) $[23.7 \% \pm 16.31$ versus $25.9 \% \pm 19.18, P=0.695]$ of spermatozoa after vitrification in 300 and $500 \mu \mathrm{l}$ volumes. For practical implications and general convenience, spermatozoa were vitrified in $300 \mu \mathrm{l}$ volumes for the purpose of the current study. The vitrification solution was prepared by dissolving HSA in double-distilled water (1\%) (Adcock Ingram, Johannesburg, South Africa), with the addition of sucrose powder (342.3 g; Sigma Aldrich, St Louis, MO, USA) to reach a $0.5 \mathrm{~m}$ sucrose concentration. Immediately after processing, the sperm suspension was diluted in a $1: 1$ ratio with the vitrification solution to reach a final sucrose concentration of $0.25 \mathrm{M}$. Straws $\left(0.5 \mathrm{ml}, \mathrm{CBS}^{\mathrm{TM}}\right)$ were marked with an asterisk $1.5 \mathrm{~cm}$ from the inner end of the cotton polyvinyl plug. This allows for a large enough air space to form inside the straw to prevent rupturing when immersed into liquid nitrogen. The vitrification and sperm solution $(300 \mu \mathrm{l})$ were aspirated into the straws. Both ends of the straws were sealed twice hermetically using an alcohol flame burner and forceps. Straws were then left at room temperature for $10 \mathrm{~min}$ and subsequently submerged horizontally into the liquid nitrogen (Isachenko et al., 2012a) and stored similarly to the conventional cryopreserved straws.

To thaw, vitrified straws were immersed into a water bath $\left(42{ }^{\circ} \mathrm{C}\right)$ for $20 \mathrm{~s}$ according to the protocol suggested by Isachenko et al. (2012a). Thereafter, straws were processed as previously described for the conventional cryopreserved samples. 


\section{Sperm motility parameters}

Motility parameters of washed spermatozoa were analysed both before freezing and post-thawing. The sperm motility parameters were analysed by computer-aided sperm analysis (CASA) using a MTG-GmbH analyser (version 5.4; MTG-MedeaLAB, Bruckberg, Germany) at 200 times magnification (Axioscope 40; Carl Zeiss, Oberkochen, Germany). For each analysis, $5 \mu \mathrm{l}$ of the sperm solution was loaded into Leja ${ }^{\mathrm{TM}}$ microchambers $\left(20 \mu \mathrm{m} ;\right.$ Leja $^{\circledR}$, Nieuw-Vennep, the Netherlands). For quality-control purposes, two separate chambers were loaded with the same spermatozoa solution, and video recordings of at least 200 cells and/or 10 random fields per chamber were carried out for $30 \mathrm{~s}$.

\section{Mitochondrial membrane potential}

The $\Delta \psi \mathrm{m}$ analysis was determined by a technique adapted from Marchetti et al. (2002). MitoTracker ${ }^{\circledR}$ Red CMXRos (Invitrogen ${ }^{\mathrm{TM}}$, Eugene, OR, USA) was used to analyse the $\Delta \psi \mathrm{m}$. A stock solution of MitoTracker ${ }^{\circledR}$ Red CMXRos (1 mm) was added to the thawed spermatozoa at a final concentration of $150 \mathrm{~nm} 10^{-6}$ and incubated $\left(37^{\circ} \mathrm{C}\right)$ for $15 \mathrm{~min}$. Hereafter, spermatozoa were washed with $2 \mathrm{ml}$ PureSperm ${ }^{\circledR}$ Wash $\left(37^{\circ} \mathrm{C}\right)$, the supernatant removed, and the sperm resuspended in $1 \mathrm{ml}$ of this medium before flow cytometry analysis (Beckman Coulter, Brea, CA, USA). MitoTracker ${ }^{\circledR}$ Red CMXRos fluorescence was detected in FL 3 channel.

The $\Delta \psi \mathrm{m}$ was abolished using $\mathrm{m}$-Chlorophenylhydrazone (mCICCP; Sigma Aldrich, St Louis, MO, USA) as a positive control. Spermatozoa were incubated with $50 \mu \mathrm{m}$ mCICCP for $15 \mathrm{~min}$, before continuing with the MitoTracker ${ }^{\circledR}$ Red CMXRos staining. For a negative control, aliquots of unstained washed spermatozoa were analysed.

\section{Detection of DNA fragmentation by the TUNEL assay}

DNA fragmentation was analysed using the APODIRECT $^{\mathrm{TM}}$ kit (BD Pharmingen ${ }^{\mathrm{TM}}$, Franklin Lakes, USA). This assay is a single-step method for labelling DNA breaks with fluorescein isothiocyanate deoxyuridine triphosphate (FITC-dUTP), followed by flow cytometry analysis (APO-DIRECTTM, 2012).

Thawed spermatozoa were fixed according to manufacturer's instruction and frozen at $-20^{\circ} \mathrm{C}$ until analysis. For fixation, thawed spermatozoa were suspended in $1 \%$ (w/v) paraformaldehyde (Sigma Aldrich, Johannesburg, South Africa) in phosphate-buffered saline (PBS, Sigma Aldrich, Johannesburg, South Africa) at a concentration of $2 \times 10^{6}$ spermatozoa $\mathrm{ml}^{-1}$. The sperm suspension was incubated on ice for $30 \mathrm{~min}$, where after spermatozoa were centrifuged and the supernatant discarded. The pellet was resuspended in the residual PBS by gentle vortexing. The sperm solution was adjusted to $1 \times 10^{6}$ spermatozoa $\mathrm{ml}^{-1}$ in $70 \%$ ice-cold alcohol. Frozen-fixed samples were thawed at room temperature and then resuspended by gently swirling the tubes, where after the samples were centrifuged for $10 \mathrm{~min}$ at $500 \mathrm{~g}$. The supernatant was aspirated, and the pellet was resuspended in $1 \mathrm{ml}$ of the supplied wash buffer. Spermatozoa were washed twice by centrifugation $(10 \mathrm{~min}$ at $500 \times \mathrm{g}$ ), and the pellet was resuspended in $50 \mu \mathrm{l}$ of the staining solution $(10 \mu \mathrm{l}$ reaction buffer, $0.75 \mu \mathrm{l}$ TdT Enzyme, $8 \mu \mathrm{L}$ FITC-dUTP and $32.25 \mu \mathrm{l}$ distilled $\mathrm{H}_{2} 0$ / assay). Samples suspended in the staining solution were incubated for $60 \mathrm{~min}$ at $37^{\circ} \mathrm{C}$, where after the solution was washed twice in $1 \mathrm{ml}$ of the supplied rinse buffer $(10 \mathrm{~min}$ at $500 \mathrm{~g})$. The supernatant was aspirated, and the pellet was resuspended in $0.5 \mathrm{ml}$ of the propidium iodide (PI)/RNase staining buffer. Thereafter, the cell suspension was incubated for $30 \mathrm{~min}$ at room temperature in the dark, after which DNA fragmentation was assessed using flow cytometry (Beckman Coulter). FITC-labelled dUTP-positive spermatozoa were detected in the FL 1 channel.

APO-DIRECT ${ }^{\mathrm{TM}}$ kits include positive and negative control cells. The control cells were stained according to the manufacturer's protocol. FITC-labelled dUTP-positive cells were measured in the FL 1 channel and PI in the FL 3 channel of the flow cytometer.

\section{Statistical analysis}

Data for the motility parameters, $\Delta \psi \mathrm{m}$ and the DNA fragmentation are reported using descriptive statistics, mean and standard deviation. This study design employed random-effects generalised least squares regression (GLS), that is, a mixed-model approach. Testing was carried out at the 0.05 level of significance using STATA RELEASE 11 statistical software (StataCorp, 2007).

\section{Results}

No statistically significant differences were observed in the total motility $(\mathrm{a}+\mathrm{b})$, rapid progressive motility (a) or velocity parameters of spermatozoa $(P>0.05)$ postthawing (see Table 1). Significantly higher $\Delta \psi \mathrm{m}$ $(11.988 \% \pm 4.326$ versus $6.581 \% \pm 1.026, P<0.001)$ and lower percentages of DNA fragmentation $(2.791 \% \pm$ 1.017 versus $3.859 \% \pm 1.381, P<0.01$ ) were found in spermatozoa cryopreserved by means of cryoprotectantfree vitrification compared with conventional cryopreservation (see Figs 1 and 2). 
Table 1 Summary of the motility and velocity parameters of spermatozoa post thawing

\begin{tabular}{|c|c|c|c|}
\hline \multirow[b]{2}{*}{ Parameter } & Vitrification & Cryopreservation & \multirow{2}{*}{$\begin{array}{l}P \text {-value } \\
(95 \% \mathrm{Cl})\end{array}$} \\
\hline & \multicolumn{2}{|c|}{ Mean (Standard deviation) } & \\
\hline Total Motility $(a+b)(\%)$ & $20.480 \%(7.399)$ & $23.900 \%(12.583)$ & $\begin{array}{l}0.181 \\
(-1.588 ; 8.428)\end{array}$ \\
\hline Rapid Progressive (a) (\%) & $11.520 \%(6.099)$ & $12.540 \%(7.160)$ & $\begin{array}{l}0.423 \\
(-1.477 ; 3.517)\end{array}$ \\
\hline $\mathrm{VCL}\left(\mu \mathrm{m} \mathrm{s}^{-1}\right)$ & $19.056 \mu \mathrm{m} \mathrm{s}^{-1}(6.469)$ & $20.646 \mu \mathrm{m} \mathrm{s}^{-1}(7.854)$ & $\begin{array}{l}0.339 \\
(-1.667 ; 4.848)\end{array}$ \\
\hline $\operatorname{VSL}\left(\mu \mathrm{m} \mathrm{s}^{-1}\right)$ & $9.857 \mu \mathrm{m} \mathrm{s}^{-1}(3.698)$ & $10.609 \mu \mathrm{m} \mathrm{s}^{-1}(4.516)$ & $\begin{array}{l}0.343 \\
(-0.804 ; 2.309)\end{array}$ \\
\hline $\operatorname{VAP}\left(\mu \mathrm{m} \mathrm{s}^{-1}\right)$ & $11.488 \mu \mathrm{m} \mathrm{s}^{-1}(4.075)$ & $12.329 \mu \mathrm{m} \mathrm{s}^{-1}(5.027)$ & $\begin{array}{l}0.385 \\
(-1.054 ; 2.735)\end{array}$ \\
\hline WOB $\left(\mu \mathrm{m} \mathrm{s}^{-1}\right)$ & $0.530(0.072)$ & $0.795(1.421)$ & $\begin{array}{l}0.349 \\
(-0.289 ; 0.819)\end{array}$ \\
\hline $\mathrm{ALH}(\mu \mathrm{m})$ & $0.503 \mu \mathrm{m}(0.118)$ & $0.548 \mu \mathrm{m}(0.140)$ & $\begin{array}{l}0.146 \\
(-0.016 ; 0.104)\end{array}$ \\
\hline
\end{tabular}

VCL, curvilinear velocity; VSL, straight line velocity; VAP, average path velocity; WOB, wobble; ALH, amplitude of lateral head displacement.

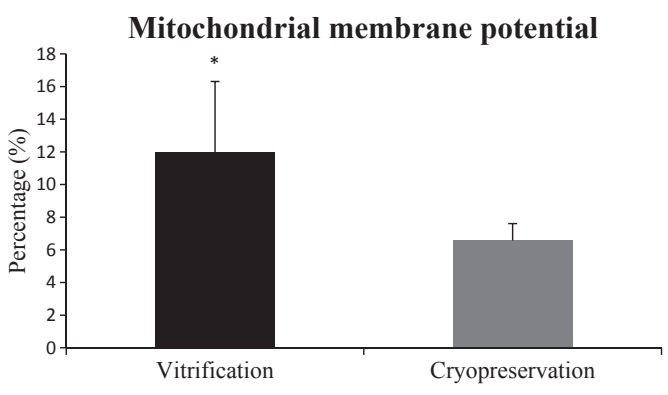

Fig. 1 Percentage of Mitotracker Red CMXROS fluorescence after freezing of spermatozoa by means of cryoprotectant-free vitrification and conventional cryopreservation $(* P<0.01)$.

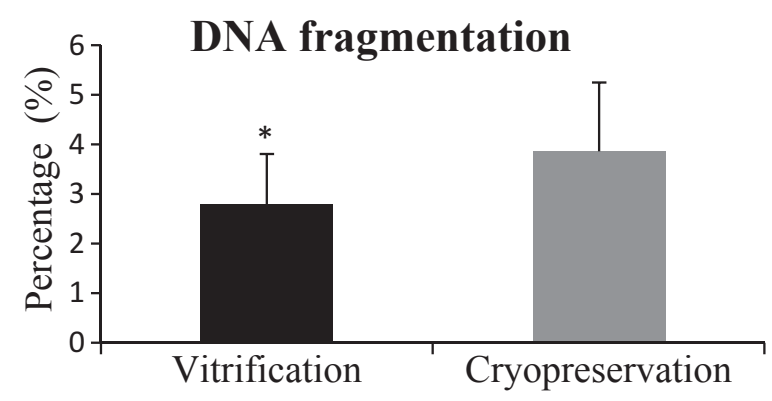

Fig. 2 Bar graph representing the percentage of DNA fragmented spermatozoa measured by means of the TUNEL (APO-DIRECT ${ }^{\mathrm{TM}}$ ) assay. $* P<0.01$

\section{Discussion}

Cryopreservation is used as a method of storing different cell types and tissues, including male and female gametes as well as embryos. As in any emerging technology, this method shows great potential, but also the need for further investigations and developments (Isachenko et al., 2012a). An advantage of vitrification, as an alternative to conventional cryopreservation, is that no cryoprotectants are needed, thus avoiding the lethal effects of cryoprotectant toxicity and osmotic damage specifically to spermatozoa (Isachenko et al., 2004a,b). Cryopreservation 'success' is measured in terms of post-thaw motility. Until now, cryopreservation has not provided complete protection, because the motility of the preserved spermatozoa decreases approximately $50 \%$ of their pre-freezing value. An even greater decrease is observed in spermatozoa from infertile patients compared with fertile patients (Donnelly et al., 2001). Therefore, the semen parameters of the samples included in this study were all above the lower reference limits recommended by the WHO (2010).

The study explored the potential and feasibility of substituting conventional cryopreservation procedures, with cryoprotectant-free vitrification for assisted reproductive procedures. Sperm motility and DNA fragmentation are considered important factors in predicting fertilisation rates (Donnelly et al., 1998; Simon \& Lewis, 2011) and were thus used as end markers in the current study. When the motility and velocity parameters of the spermatozoa were compared after conventional cryopreservation and vitrification, no statistically significant differences were observed. The percentages of rapid progressively motile (a) and total motile $(\mathrm{a}+\mathrm{b})$ spermatozoa after cryopreservation were $12.54 \%$ and $23.9 \%$ $(P>0.05)$, respectively, compared with $11.52 \%$ and $20.48 \%(P>0.05)$ for vitrified spermatozoa. Concurrent results regarding sperm motility (progressive motility [19.45\%] and total motility [25.4\%]) were reported 
by Moskovtsev et al. (2012) using a similar methodology. Significantly higher percentages of motile spermatozoa after cryoprotectant-free vitrification were, however, described by Isachenko et al. (2011) and Sánchez et al. (2011). A similar vitrification protocol was followed by Isachenko et al. (2011) and the case study by Sánchez et al. (2011) utilised a different protocol when compared to the current study. In addition, a decrease in the equilibration time (10 min used in this study) could prevent further osmotic damage to the spermatozoa, possibly resulting in higher post-thaw sperm motility and recovery rates.

Spermatozoa preserved by means of the cryoprotectant-free vitrification technique presented with significantly $(P<0.001)$ higher $\Delta \psi \mathrm{m}$, compared with the conventional cryopreservation technique. The $\Delta \psi \mathrm{m}$ of post-thaw vitrified spermatozoa was also determined by Isachenko et al. (2008, 2012b) and Sánchez et al. (2011). Results obtained in this study are considerably lower than results reported by the latter authors. The different carrying devices as well as vitrification volumes can possibly contribute to the differences in $\Delta \psi \mathrm{m}$ values observed. Reductions in the $\Delta \psi \mathrm{m}$ are often associated with a decrease in sperm motility (Thornberry \& Lazebnik, 1998). The percentage of motile spermatozoa recovered post-thawing was significantly lower than the results reported by Isachenko et al. (2008, 2012b), further explaining the difference in the mitochondrial potential found post-thawing. Motility results obtained from this current study do not necessarily explain this phenomenon, because no difference was observed in the sperm motility and velocity parameters between the two techniques. The decrease in the $\Delta \psi \mathrm{m}$ could possibly be considered as a feature of cell death, where the identification of mitochondrial dysfunctions is early markers of programmed cell death in somatic cells (Green \& Reed, 1998; Thornberry \& Lazebnik, 1998). A reduction in the $\Delta \psi \mathrm{m}$ defines an early stage of apoptosis preceding other manifestation processes such as DNA fragmentation and reactive oxygen species production (Kroemer et al., 1997; Marchetti et al., 2002). DNA strand breaks typically occur during the final stages of cell death. Results from the TUNEL assay (APO-DIRECT ${ }^{\mathrm{TM}}$ ) also confirmed this theory, as significantly $(P<0.01)$ higher percentages of DNA-fragmented spermatozoa were found after conventional cryopreservation in comparison with cryoprotectant-free vitrification. Conversely, Moskovtsev et al. (2012) reported no significant difference in the DNA fragmentation after preserving spermatozoa by means of vitrification versus conventional cryopreservation.

Results from this study indicated that after thawing only one straw $(300 \mu \mathrm{l})$ of spermatozoa preserved either by means of conventional cryopreservation or cryoprotectant- free vitrification, $1.92 \times 10^{6}( \pm 0.557)$ and $1.71 \times 10^{6}$ $( \pm 0.428)$ rapid progressively motile (a) spermatozoa were recovered respectively. Intrauterine insemination (IUI) can be promoted as a first-line therapy in cases with at least $1 \times 10^{6}$ motile spermatozoa after washing for successful fertilisation. In circumstances with an inseminating motile count of less than $1 \times 10^{6}$, IUI can still be performed, provided the morphology score is $\geq 4 \%$ (Ombelet et al., 1997). Therefore, these methods are comparable, and either can be implemented for the storage of spermatozoa to be used for future ART procedures. Vitrification of spermatozoa provides a simpler, faster, more cost-effective alternative to conventional cryopreservation methods. Laboratories with limited resources and budget constraints can easily utilise this technique as no specialised or extra freezing equipment is needed. In addition, this technique does not require any expensive cryopreservation mediums and can easily be performed in any laboratory.

\section{Acknowledgements}

This study was funded by the Research Committee of the Faculty of Health Sciences (RESCOM), University of Pretoria, Merck Serono (Pty) Ltd and the National Research Foundation. The authors would like to acknowledge Prof P. Becker from the Medical Research Counsel (MRC) for statistical guidance and Ms B. English (UP) for language consultation.

\section{References}

APO-DIRECTTM (2012) [product insert]. BD PharmingentM, Franklin Lakes, USA.

Donnelly ET, Lewis SEM, McNally JA, Thompson W (1998) In vitro fertilization and pregnancy rates: The influence of sperm motility and morphology on IVF outcome. Fertil Steril 70:305-314.

Donnelly ET, Steele EK, McClure N, Lewis SE (2001) Assessment of DNA integrity and morphology of ejaculated spermatozoa from fertile and infertile men before and after cryopreservation. Hum Reprod 16:1191-1199.

Green DR, Reed JC (1998) Mitochondria and apoptosis. Science 28:1309-1312.

Isachenko E, Isachenko V, Katkov II, Dessole S, Nawroth F (2003) Vitrification of mammalian spermatozoa in the absence of cryoprotectants: From past practical difficulties to present success. Reprod Biomed Online 6:191-200.

Isachenko E, Isachenko V, Katkov II, Rahimi G, Schöndorf T, Mallmann P, Dessole S, Nawroth F (2004a) DNA integrity and motility of human spermatozoa after standard slow freezing versus cryoprotectant-free vitrification. Hum Reprod 19:932-939.

Isachenko V, Isachenko E, Katkov II, Montag M, Dessole S, Nawroth F, van der Ven H (2004b) Cryoprotectant-free 
cryopreservation of human spermatozoa by vitrification and freezing in vapor: effect on motility, DNA integrity, and fertilization ability. Biol Reprod 71:1167-1173.

Isachenko $\mathrm{V}$, Isachenko $\mathrm{E}$, Montag $\mathrm{M}$, Zaeva $\mathrm{V}$, Krivokharchenko I, Nawroth F, Dessole S, Katkov II, van der Ven H (2005) Clean technique for cryoprotectant-free vitrification of human spermatozoa. Reprod Biomed Online 10:350-354.

Isachenko E, Isachenko V, Weiss JM, Kreienberg R, Katkov II, Schulz M, Lulat AGMI, Risopatron MJ, Sanchez R (2008)

Acrosomal status and mitochondrial activity of human spermatozoa vitrified with sucrose. Hum Reprod 136:167-173.

Isachenko V, Maettner R, Petrunkina AM, Mallmann P, Rahimi G, Sterzik K, Sanchez R, Risopatron J, Damjanoski I, Isachenko E (2011) Cryoprotectant-free vitrification of human spermatozoa in large (up to $0.5 \mathrm{~mL}$ ) volume: a novel technology. Clin Lab 57:643-650.

Isachenko E, Mallmann P, Rahimi G, Risopatron J, Schulz M, Isachenko V, Sanchez R (2012a) Vitrification technique-new possibilities for male gamete low-temperature storage. In: Current Frontiers in Cryobiology. Katkov I (ed.). InTech, Croatia, pp. 41-76.

Isachenko V, Isachenko E, Petrunkina AM, Sanchez R (2012b) Human spermatozoa vitrified in the absence of permeable cryoprotectants: birth of two healthy babies. Reprod Fertil Dev 24:323-326.

Kroemer G, Zamzami N, Susin SA (1997) Mitochondrial control of apoptosis. Immunol Today 18:44-51.

Marchetti C, Obert G, Defossez A, Formstecher P, Marchetti P (2002) Study of mitochondrial membrane potential, reactive oxygen species, DNA fragmentation and cell viability by flow cytometry in human sperm. Hum Reprod 17:12571265.

Moskovtsev SI, Lulat AGM, Librach CL (2012)

Cryopreservation of human spermatozoa by vitrification vs. slow freezing: Canadian experience. In: Current frontiers in Cryobiology. Katkov II (ed.). InTech, Croatia, pp. 77-101.

Ombelet W, Vandeput H, Van de Putte G, Cox A, Janssen M, Jacobs P, Bosman E, Steeno O, Kruger T (1997) Intrauterine insemination after ovarian stimulation with clomiphene citrate: predictive potential of inseminating motile count and sperm morphology. Hum Reprod 12:1458-1463.

Sánchez R, Isachenko V, Petrunkina A, Risopatrón J, Schulz M, Isachenko E (2011) Live birth after intrauterine insemination with spermatozoa from an oligo-asthenozoospermic patient vitrified without permeable cryoprotectants. J Androl 33:559-562.

Simon L, Lewis SEM (2011) Sperm DNA damage or progressive motility: which one is the better predictor of fertilization in vitro? Syst Biol Reprod Med 57:133-138.

StataCorp (2007) Stata Statistical Software: Release 10, 10th edn. StataCorp LP, College Station.

Thornberry NA, Lazebnik Y (1998) Caspases: Enemies within. Science 281:1312-1316.

Vutyavanich T, Piromlertamorn W, Nunta S (2010) Rapid freezing versus slow programmable freezing of human spermatozoa. Fertil Steril 93:1921-1928.

World Health Organization (2010) WHO Laboratory Manual for the Examination and Processing of Human Semen, 5th edn. Cambridge University Press, Cambridge. 